\title{
Performance Enhancement by the Design of Flash Controller for $x 8$ NAND Flash Memory Devices
}

\author{
Pramod T. Talole \\ M.E. (Digital Electronics) \\ S.S.G.M.C.E., Shegaon, Dist: Buldana [MS]
}

\author{
Sarita T. Sawale \\ M.E. (CSE) \\ Govt. College of Engg. Aurangabad [MS]
}

\begin{abstract}
The available flash devices are NAND flash and NOR flash memory devices. Each device is also divided with data width into x8 / x16 NAND flash memory and x8 / x16 NOR flash memory. We are going to implement Flash Controller which is the interface between $\mathrm{x} 8 \mathrm{NAND}$ flash device and the CPU/processor. The same concept will be used for other devices like $x 8$ NOR also. Normal interface is between one $\mathrm{x} 8$ NAND flash device and CPU or Processor. This paper proposed a design to interface eight $\mathrm{x} 8$ NAND flash devices with CPU/processor. The main feature of the implementation is parallel write operation of eight $\mathrm{x} 8$ NAND flash devices. As the numbers of flash devices are 8, it requires dedicated DMA buffers for each x8 NAND flash device. Also to support parallel writes, it is required to read the data of a next DMA write from DRAM while current NAND flash write is in progress. Hence DMA engine is divided into two parts - Flash Side DMA Engine (DMA) and Host Side DMA Engine (HDE). These DMA engines are used to control the above operation.
\end{abstract}

\section{Keywords}

Design

\section{INTRODUCTION}

The flash interface unit is designed for system on chip (SoC) applications where flash memory acts as the main non volatile storage device. The need for non-volatile flash memory is increasing as many new applications require a system with no hard disks. Flash memory provides storage which is rugged, requires low power, and comes in a small and light form factor. The flash interface unit provides basic flash commands which can be used by the main CPU to access data in the flash memory. Since the flash memory contains information to initiate the boot process, it is assumed that the flash memory is non-removable. Since the flash memory acts as the main storage device, the main CPU should be able to read and write files like any generic file system. However, flash memory does not follow the standard $\mathrm{read} / \mathrm{write}$ interface. Write access to flash memory is only through programs which change bits in memory from one to zero. In order to change bits in memory from zero to one, flash memory supports erases which sets blocks of memory to all ones.

\section{MOTIVATION}

The flash interface unit has been designed primarily to support DMP10, DMP20, and SC25. DMP10 and DMP20 are high end 2D/3D graphics and multimedia processors designed primarily for digital television, car navigation and pachinko applications. SC25 is an ultra low power $2 \mathrm{D} / 3 \mathrm{D}$ graphics and multimedia processor designed primarily for mobile applications.

In order to support multimedia applications, the flash interface unit has been optimized for large block reads and writes. This interface must satisfy the bandwidth requirements required to transfer video and audio streams from flash memory, with special attention to write bandwidth to minimize downloading time. To minimize main CPU interaction, the flash interface unit supports DMA transfers from flash memory to system DRAM memory.

In SoC applications, the amount of on-chip ROM memory dedicated to booting is very limited. As a result, most or all of the boot information is typically stored in flash memory. A number of partitions for the boot loader code, the compressed root application or the flash file system are created in the flash memory.

Table 1. Properties

\begin{tabular}{|c|c|c|c|}
\hline Devices & NOR & Small NAND & Large NAND \\
\hline $\begin{array}{c}\text { Approximate } \\
\text { cost }\end{array}$ & $\$ 0.30 / \mathrm{MB}$ & $\$ 0.08 / \mathrm{MB}$ & $\$ 0.06 / \mathrm{MB}$ \\
\hline Density/device & $\begin{array}{l}16 \mathrm{Mb} \text { to } \\
128 \mathrm{Mb}\end{array}$ & $64 \mathrm{Mb}$ to $2 \mathrm{~Gb}$ & $1 \mathrm{~Gb}$ to $8 \mathrm{~Gb}$ \\
\hline $\begin{array}{l}\text { Memory } \\
\text { Access }\end{array}$ & $\begin{array}{c}\text { Random } \\
\text { Access } \\
\text { XIP Support }\end{array}$ & $\begin{array}{l}\text { Page Access } \\
\text { No XIP } \\
\text { Support }\end{array}$ & $\begin{array}{l}\text { Page Access } \\
\text { No XIP } \\
\text { Support }\end{array}$ \\
\hline Page Size & $\mathrm{N} / \mathrm{A}$ & $\begin{array}{c}512 \text { bytes/16 } \\
\text { byte spare }\end{array}$ & $\begin{array}{c}2048 \text { bytes/ } 64 \\
\text { byte spare }\end{array}$ \\
\hline Block Size & $8 \mathrm{~KB} / 64 \mathrm{~KB}$ & $\begin{array}{c}16 \mathrm{~KB}(32 \\
\text { pages) }\end{array}$ & $\begin{array}{c}128 \mathrm{~KB} \text { (64 } \\
\text { pages) }\end{array}$ \\
\hline $\begin{array}{c}\text { Read Access } \\
\text { Time }\end{array}$ & $\begin{array}{c}65 \mathrm{~ns} \text { (first } \\
\text { byte) } \\
25 \mathrm{~ns} \text { (next } 7 \\
\text { bytes) }\end{array}$ & $\begin{array}{c}\text { 50ns (cycle } \\
\text { time) } \\
\text { 10us (page } \\
\text { time) }\end{array}$ & $\begin{array}{l}\text { 30ns (cycle } \\
\text { time) } \\
25 \text { us (page } \\
\text { time) }\end{array}$ \\
\hline Program Time & 4us per byte & $\begin{array}{c}\text { 200us per } \\
\text { page }\end{array}$ & $\begin{array}{c}\text { 200us per } \\
\text { page }\end{array}$ \\
\hline $\begin{array}{c}\text { Block Erase } \\
\text { Time }\end{array}$ & 0.7 to $1.6 \mathrm{sec}$ & $2 \mathrm{~ms}$ & $2 \mathrm{~ms}$ \\
\hline $\begin{array}{c}\text { Shipped with } \\
\text { Bad Blocks }\end{array}$ & No & Yes & Yes \\
\hline $\begin{array}{c}\text { ECC } \\
\text { Recommended } \\
\end{array}$ & No & Yes & Yes \\
\hline
\end{tabular}




\section{PERFORMANCE REQUIREMENT}

Bandwidth for NAND, the peak read bit rate is

$$
=\frac{n_{p} w}{t_{w c}\left(n_{c}+n_{a}\right)+t_{r}+t_{r c} n_{p}}
$$

With

$n_{p}$ the number of data accesses in a page

$w$ the width of the data bus

$n_{c}$ the number of commands to initiate a full page read, typically one.

$n_{a}$ the number of address cycles to initiate a full page read

$t_{w c}$ the write cycle time, used to send command and address to the flash

$t_{r}$ the retrieval time to transfer the data into the page buffer (also called register for NAND)

$t_{r c}$ the read cycle time per data access

\subsection{Small Flash Bandwidth (x8 NAND)}

DMP10 can be supported by a small NAND flash x8/x16. 6 Mbps write and $10 \mathrm{Mbps}$ read. NAND Flash takes 10 us to access a page, and 50 ns to read each byte/word. Max x8 device FlashReadBW $=512$ bytes $/(10$ us $+512 * 50 \mathrm{~ns})=14.4 \mathrm{MB} / \mathrm{sec}$. Max x16 device FlashReadBW $=256$ words $/(10 \mathrm{us}+256 * 50 \mathrm{~ns})=$ $22.5 \mathrm{MB} / \mathrm{sec}$. NAND Flash takes 200 us to program a page, and $50 \mathrm{~ns}$ to write each byte/word. Max x8 device FlashWriteBW = 512 bytes/ $(200 \mathrm{us}+512 * 50 \mathrm{~ns})=2.27 \mathrm{MB} / \mathrm{sec}$. Max x16 device FlashWriteBW $=256$ words $/(200 \mathrm{us}+256 * 50 \mathrm{~ns})=2.41 \mathrm{MB} / \mathrm{sec}$.

\subsection{Large NAND Flash Bandwidth}

DMP20 can be supported by a large NAND flash x8/x16. 19 Mbps write and $38 \mathrm{Mbps}$ read. NAND Flash takes 25 us to access a page, and $30 \mathrm{~ns}$ to read each byte/word. Max $\mathrm{x} 8$ device FlashReadBW $=2048$ bytes $/(25$ us $+2048 * 30 \mathrm{~ns})=23.7 \mathrm{MB} / \mathrm{sec}$. Max x16 device FlashReadBW $=1024$ words/ (25us + $1024 * 30 \mathrm{~ns})=36.8 \mathrm{MB} / \mathrm{sec}$. NAND Flash takes 200 us to program a page, and $30 \mathrm{~ns}$ to write each byte/word. Max x8 device FlashWriteBW $=2048$ bytes/ $(200 \mathrm{us}+2048 * 30 \mathrm{~ns})=7.83$ MB/sec. Max x16 device FlashWriteBW $=1024$ words/ (200us + $1024 * 30 \mathrm{~ns})=8.88 \mathrm{MB} / \mathrm{sec}$.

\section{PARALlel WRITE OF MULTIPLE NAND FLASH DEVICES.}

The previous rate requirements were for one device. When multiple external devices are present, the aggregate write performance can be increased by concurrent use of multiple external devices. This can be done efficiently without resorting to full parallelization as explained below.

The following tables show numerical values for the BW of different operations. The table also shows a duty cycle, defined as the ratio between the amount of cycles where chip select is asserted to the total operation time and how many devices can operate on the bus without the bus becoming the bottleneck.
Table 2. Samsung K9xxG08UxM: 8 bits data bus, $N p=2 k+$ 64, worst case $T_{P R O G}$ and $T_{B E R S}$

\begin{tabular}{|c|c|c|c|c|c|}
\hline Operation & $\begin{array}{c}\text { T bus } \\
\text { (ns) }\end{array}$ & $\begin{array}{c}\mathbf{T} \\
\text { operation } \\
(\mathbf{n s})\end{array}$ & $\begin{array}{c}\text { BW } \\
(\mathbf{M b} / \mathbf{s})\end{array}$ & $\begin{array}{c}\text { Duty } \\
\text { Cycle }\end{array}$ & $\begin{array}{c}\text { Max } \\
\text { Devices }\end{array}$ \\
\hline Erase & 225 & $\begin{array}{c}40,000,22 \\
5\end{array}$ & 27.0 & $0.00 \%$ & $\begin{array}{c}177,77 \\
8\end{array}$ \\
\hline Write & $\begin{array}{c}53,05 \\
0\end{array}$ & 753,050 & 22.4 & $7.04 \%$ & 14 \\
\hline Read & $\begin{array}{c}52,97 \\
5\end{array}$ & 72,825 & 232.0 & $\begin{array}{c}72.74 \\
\%\end{array}$ & 1 \\
\hline
\end{tabular}

Table 3. Same device, Samsung K9xxG08UxM, but typical case $T_{P R O G}$ and $T_{B E R S}$

\begin{tabular}{|c|c|c|c|c|c|}
\hline Operation & $\begin{array}{c}\text { T bus } \\
\text { (ns) }\end{array}$ & $\begin{array}{c}\mathbf{T} \\
\text { operation } \\
(\mathbf{n s})\end{array}$ & $\begin{array}{c}\text { BW } \\
(\mathbf{M b} / \mathbf{s})\end{array}$ & $\begin{array}{c}\text { Duty } \\
\text { Cycle }\end{array}$ & $\begin{array}{c}\text { Max } \\
\text { Devices }\end{array}$ \\
\hline Erase & 225 & $\begin{array}{c}30,000,22 \\
5\end{array}$ & 36.0 & $0.00 \%$ & 133,334 \\
\hline Write & 53,050 & 253,050 & 66.8 & $\begin{array}{c}20.96 \\
\%\end{array}$ & $\begin{array}{c}4 \text { (almost } \\
5)\end{array}$ \\
\hline Read & 52,975 & 72,825 & 232.0 & $\begin{array}{c}72.74 \\
\%\end{array}$ & 1 \\
\hline
\end{tabular}

Table 4. Toshiba TC58NVG0S3AFT05: 8 bits data bus, $\mathrm{Np}=$ $2 k+64$, worst case $T_{P R O G}$ and $T_{\text {BERS }}$

\begin{tabular}{|c|c|c|c|c|c|}
\hline Operation & $\begin{array}{c}\text { T bus } \\
\text { (ns) }\end{array}$ & $\begin{array}{c}\mathbf{T} \\
\text { operation } \\
\text { (ns) }\end{array}$ & $\begin{array}{c}\text { BW } \\
\text { (Mb/s) }\end{array}$ & $\begin{array}{c}\text { Duty } \\
\text { Cycle }\end{array}$ & $\begin{array}{c}\text { Max } \\
\text { Devices }\end{array}$ \\
\hline Erase & 450 & $\begin{array}{c}80,000,45 \\
0\end{array}$ & 13.5 & $0.00 \%$ & 177,778 \\
\hline Write & $\begin{array}{c}106,10 \\
0\end{array}$ & 806,100 & 21.0 & $\begin{array}{c}13.16 \\
\%\end{array}$ & 7 \\
\hline Read & $\begin{array}{c}105,95 \\
0\end{array}$ & 130,650 & 129.3 & $\begin{array}{c}81.09 \\
\%\end{array}$ & 1 \\
\hline
\end{tabular}

Table 5. Same device, Toshiba TC58NVG0S3AFT05, but typical case $T_{P R O G}$ and $T_{B E R S}$

\begin{tabular}{|c|c|c|c|c|c|}
\hline $\begin{array}{c}\text { Operatio } \\
\mathbf{n}\end{array}$ & $\begin{array}{c}\text { T bus } \\
\text { (ns) }\end{array}$ & $\begin{array}{c}\mathbf{T} \\
\text { operatio } \\
\mathbf{n}(\mathbf{n s})\end{array}$ & $\begin{array}{c}\mathbf{B W} \\
\mathbf{( M b} / \mathbf{s} \\
\text { ) }\end{array}$ & $\begin{array}{c}\text { Duty } \\
\text { Cycle }\end{array}$ & $\begin{array}{c}\text { Max } \\
\text { Devices }\end{array}$ \\
\hline Erase & 450 & $\begin{array}{c}40,000,4 \\
50\end{array}$ & 27.0 & $0.00 \%$ & 88,889 \\
\hline Write & 106,100 & 306,100 & 55.2 & $\begin{array}{c}34.66 \\
\%\end{array}$ & $\begin{array}{c}2 \\
\text { (almost } \\
3)\end{array}$ \\
\hline Read & 105,950 & 130,650 & 129.3 & $\begin{array}{c}81.09 \\
\%\end{array}$ & 1 \\
\hline
\end{tabular}

When $\mathrm{N}$ devices are present, the peak rate can trivially be $\mathrm{N}$ times the above rate by providing independent channels to each device. However, if maximizing the write bandwidth is the goal, it is possible to increase the write bandwidth by a factor $\mathrm{M}$ using one 
bus and $\mathrm{M}$ chip select signals. This principle is shown in the figure 1 .

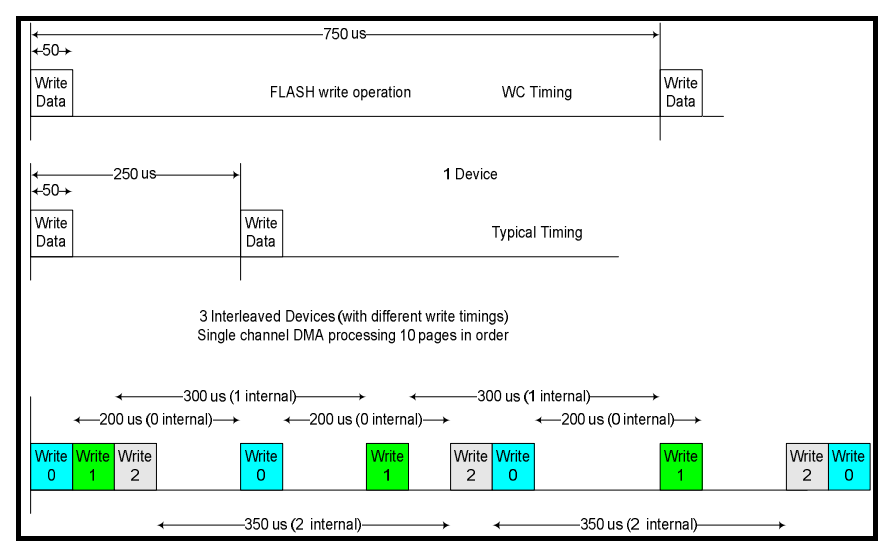

Figure 1. Flash writes operation.

The next table shows the peak write bandwidth in function of the number of devices on one data bus, bold value identify configurations where the bus is the bottleneck, not the devices. These values ignore the effects of collisions.

Table 6. Peak Writes Bandwidth

\begin{tabular}{|c|c|c|c|c|}
\hline $\begin{array}{c}\text { Device } \\
\text { type }\end{array}$ & \multicolumn{2}{|c|}{$\begin{array}{c}\text { Samsung } \\
\text { K9xxG08UxM }\end{array}$} & \multicolumn{2}{c|}{$\begin{array}{c}\text { Toshiba } \\
\text { TC58NVG0S3AFT05 }\end{array}$} \\
\hline Device(s) & $\begin{array}{c}\text { Worst } \\
\text { case }\end{array}$ & $\begin{array}{c}\text { Typical } \\
\text { case }\end{array}$ & Worst case & $\begin{array}{c}\text { Typical } \\
\text { case }\end{array}$ \\
\hline 1 & 22.4 & 66.8 & 21.0 & 55.2 \\
\hline 2 & 44.8 & 133.6 & 42.0 & 110.4 \\
\hline 3 & 67.2 & 200.4 & 63.0 & $\mathbf{1 6 0 . 0}$ \\
\hline 4 & 89.6 & 267.2 & 84.0 & $\mathbf{1 6 0 . 0}$ \\
\hline 5 & 112.0 & $\mathbf{3 2 0 . 0}$ & 105.0 & $\mathbf{1 6 0 . 0}$ \\
\hline 6 & 134.4 & $\mathbf{3 2 0 . 0}$ & 126.0 & $\mathbf{1 6 0 . 0}$ \\
\hline 7 & 156.8 & $\mathbf{3 2 0 . 0}$ & 147.0 & $\mathbf{1 6 0 . 0}$ \\
\hline 8 & 179.2 & $\mathbf{3 2 0 . 0}$ & 160.0 & $\mathbf{1 6 0 . 0}$ \\
\hline
\end{tabular}

Based on these values and the requirements, the flash interface unit will operate with one NAND flash interface, supporting 8 chip select signals. To limit the number of signals, only one ready signal is used per interface and the status byte is polled to detect when a write operation is finished. The polling operation has low priority; especially polling doesn't take place if the DMA is transferring data to another device. Only DMA program operations are operated in this fashion.

\section{FLASH CONTROLLER}

The Flash Controller mainly comprises of toggle, DMA Engine and Flash Interface block.
Figure 2. NAND Flash Controller

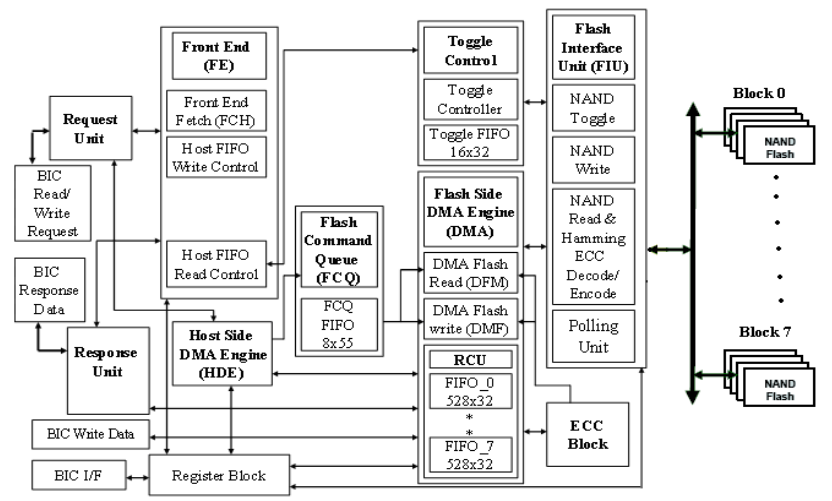

\subsection{Front-End Block}

The Flash interface unit implements a small number of methods. Hence fetch, decode, validate, notify, package generation and emitter functionality is implemented in this block. Methods processed by DMA engine and toggle control are specified in class file. A Perl script will be written to generate verilog and Fmodel files. Front-end emits a package (DMA operation) on one bus to the DMA engine and bundles (toggle commands) on another bus to the toggle control block. Front-end waits for acknowledge from the engines that consume the packages before sending a new package or bundle.

\subsubsection{Host}

This block implements a 64-entry FIFO $(64 \times 64)$ to store the commands that are fetched from memory. Commands are fetched by performing DMA operation (getPtr/putPtr). The flushing semantics impacts the combination of FIFO in the host and the rest of the command buffer in memory.

The host fetches commands from memory when getPtr and putPtr are different and fetch is enabled. In particular, $\mathrm{S} / \mathrm{W}$ is allowed to modify the putPtr at any time.

The host will forward commands to the execution engine in the following way. When the FIFO is not empty, the host checks the command at the head of FIFO and operates. If a block is currently active and the next command is not for the same block, wait the block becomes inactive before attempting to issue the next command. This insures that toggle and DMA are never active simultaneously. It also insures that an interrupt is only generated after a DMA transfer is fully completed.

An inactive block is assumed to be able to accept any command. An active block indicates provides an indication to the host about which commands it is able to accept (flow control). This is especially needed to insure parallel operation of the DMA write; the DMA provides at least 8 flags to the host, each flag indicating that the channel is able to accept a new command.

\subsubsection{Notify}

Notification is supported via flags in $\mathrm{H} / \mathrm{W}$ registers and by interrupts. In the list of events previously notified, the DMP10 alternate support is shown in italics. 
A 32-bit free running counter (wraps at $\sim 43 \operatorname{secs}$ at $100 \mathrm{MHz}$ ) is maintained to timestamp the notification messages. Notification is sent to software on the occurrence of the following events:

Upon reception of a notify command (P1). Only an interrupt command as $P O$.

Upon detection of a correctable error when connected to a NAND flash (P1). Error captured in error log register as $\mathrm{P} 0$.

Upon detection of an uncorrectable error when connected to a NAND flash (P1). Error captured in error log register as $P 0$.

Upon detection of programming errors or read errors by the flash device (P1). Only timeout interrupt as PO.

Upon reception of a DMA command that overlaps multiple pages in a NAND flash device. Only interrupt during execution of DMA command as $P O$.

Upon reception of a DMA command that exceeds the supported size of the flash device. Only interrupt during execution of DMA command as $P 0$.

\subsubsection{Request Arbiter}

Arbitrates between the three memories requesters: Front-end fetch, DMA engine and Notify block (P1). The order of priority in case of multiple requests arriving in the same clock is: Notify followed by Front-end fetch followed by DMA.

\subsection{DMA Engine}

DMA engine supports the following features:

Transfer size limited to $2 \mathrm{~KB}$ maximum for large page NAND, and 512B for small page NAND flash devices.

Breaks down DMA transfers into smaller transfers depending on the size of bursts supported by the interface. The bursts should be as long as possible (64 bytes).

Support multiple outstanding read and write messages towards the memory controller so that memory controller can utilize memory bandwidth efficiently.

All data transfers are multiple of 32 bits and naturally aligned for ease of implementation.

Supports multiple write and erase program in parallel.

The DMA starts transferring data upon reception of the DMA packet (consisting of command type, source address, destination address and size) from the front-end.

DMA inside a NAND has some specific characteristics: The flash address is used to calculate a start page and the target device, the address passed to the NAND is a combination of page address and offset in the page. When needed the DMA interact with the ECC block to either generate the ECC bytes to program in the spare area or to correct data present in the data buffer. ECC errors are logged. For correctable errors, the correct amount of data is transferred into memory.

The DMA processes commands sequentially as fast as possible and overlaps DMA write and erase commands for best performance.

\subsection{DMA Buffer}

The data buffer is implemented as 8 blocks, each of $(512+16) \mathrm{x}$ 32 SRAM, managing the flow of information between DMA, enhanced ECC block and the external flash devices. The data buffer is statically provisioned with $2 \mathrm{~K}+64$ bytes (one large page including spare area) per external flash device. The ECC block needs access to the DMA buffer, but only one of DMA or ECC is allowed access to one of the page buffer at any one time.

The normal sequence of operation is begin from DMA command results in data transfer into one of the DMA buffer, either from external DRAM or from flash. DMA pass the DMA buffer to ECC if needed, ECC either encodes or decodes the codeword. ECC signals to DMA that processing is done; control of DMA buffer is given back to DMA. DMA finishes the DMA command, transferring DMA buffer to either flash or external DRAM RAM Parameters like single cycle read and write access, byte enables for write and shared read/write port.

\section{NAND INTERFACE WRITE BLOCK}

The NAND interface write block is a part of DMA page program operation. The complete DMA page program operation is done through the NAND interface write block and NAND interface polling block. The write FSM is active up to the page program confirm command (10h) which initiates the programming process/cycle. The NAND interface write block contains two write state machines. Main write state machine is used to controls the main field area and spare field area. NAND write sub-state machine is used to control the exact write process both for main field area as well as spare field area and is dependent on write state machine [3, 4].

\section{EXPERIMENTAL SETUP AND RESULTS}

In this design, we have studied the logic implementation ideas used in the NAND - Flash 1.2-Flyer document from Arasan. In which the NAND flash interface provides an 8-bit or 16-bit interface to the flash memories. A total of 4 memory banks and 8 flash memories per bank are supported. Each memory bank provides up to 8 chip selects signals. The interface supports a maximum of 64 Gbytes of NAND flash memory [12].

From this concept the controller designs for the 8-bit interface to the NAND Flash memory to support parallel write operation. The NAND flash Verilog model-MT29F (Micron Technology, Inc.) is used for verification and for simulation- Modelsim SE 5.8 is used with $2.8 \mathrm{GHz}$ Pentium IV processor, $512 \mathrm{MB}$ RAM running Windows XP system $[10,11]$.

\subsection{PAGE PROGRAM}

The device is programmed basically on a page basis, but it does allow multiple partial page programming of a byte/word or consecutive bytes/words up to 528(X8 device) or 264(X16 device), in a single page program cycle. The number of consecutive partial page programming operation within the same page without an intervening erase operation should not exceed 2 for main array and 3 for spare array. The addressing may be done in any random order in a block. A page program cycle consists of a serial data loading period in which up to 528 bytes(X8 device) or 264 words(X16 device) of data may be loaded into the page 
register, followed by a non-volatile programming period where the loaded data is programmed into the appropriate cell. The serial data loading period begins by inputting the Serial Data Input command $(80 \mathrm{~h})$, followed by the three cycle address input and then serial data loading. The words other than those to be programmed do not need to be loaded. The Page Program confirms command (10h) initiates the programming process. Writing $10 \mathrm{~h}$ alone without previously entering the serial data will not initiate the programming process. The internal write controller automatically executes the algorithms and timings necessary for program and verify, thereby freeing the system controller for other tasks. Once the program process starts, the Read Status Register command may be entered, with RE and CE low, to read the status register. The system controller can detect the completion of a program cycle by monitoring the R/B output, or the Status bit (I/O 6) of the Status Register. Only the Read Status command and Reset command are valid while programming is in progress. When the Page Program is complete, the Write Status Bit (I/O 0) may be checked (Figure 5). The internal write verify detects only errors for "1"s that are not successfully programmed to "0"s. The command register remains in Read Status command mode until another valid command is written to the command register $[3,4]$.

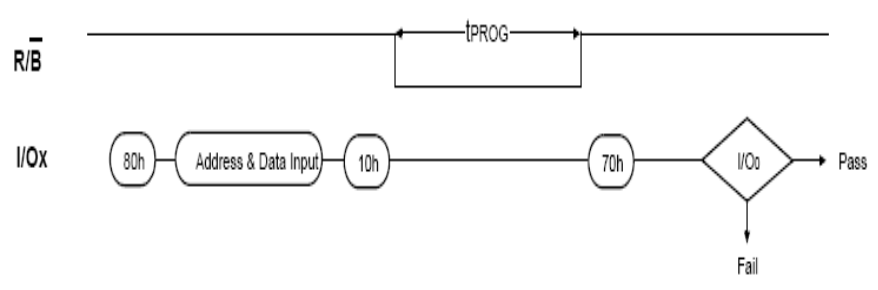

Fig 4: Program Operation

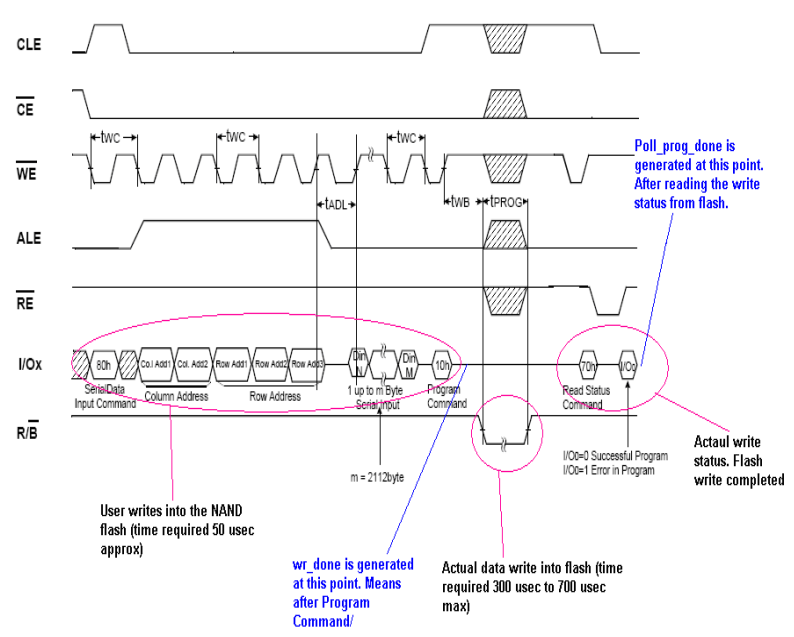

Fig.5: Page Write Cycle

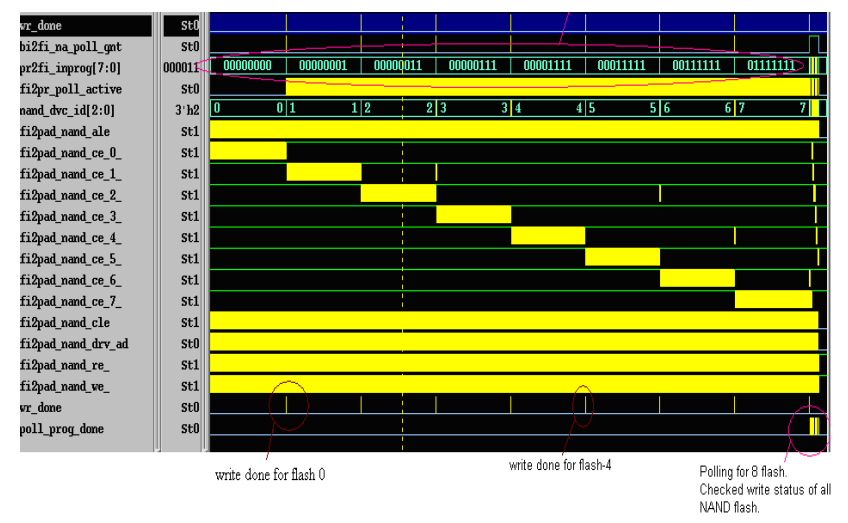

Fig.6: 8-Flash Parallel Programmed

8 NAND flash are programmed by Parallel Write method. The actual flash data write time is more, so the same time is utilized to program the next flash. First priority is given to the user to write the flash and second priority is given for polling to check the write status of each flash.

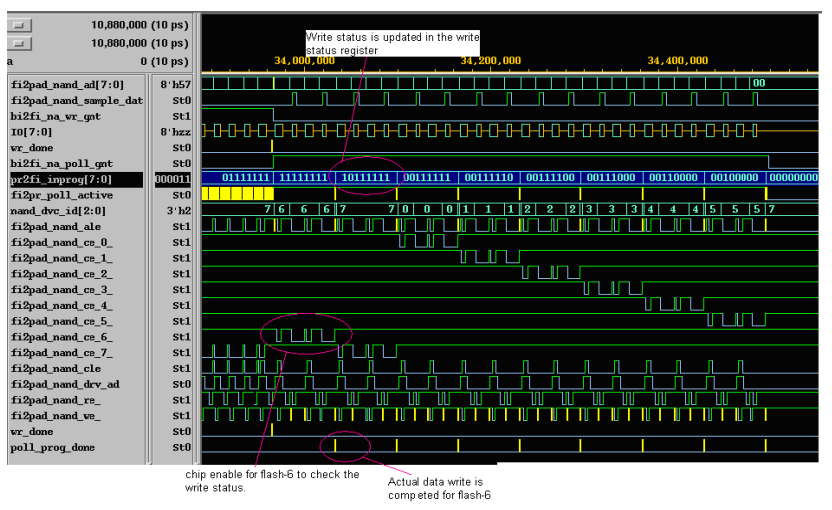

Fig. 7: 8-Flash Parallel Programmed Polling Cycles

The actual data time into flash is 300 microseconds to 700 microseconds. In this case, we need less than 50usec to check the actually write completion status of each flash.

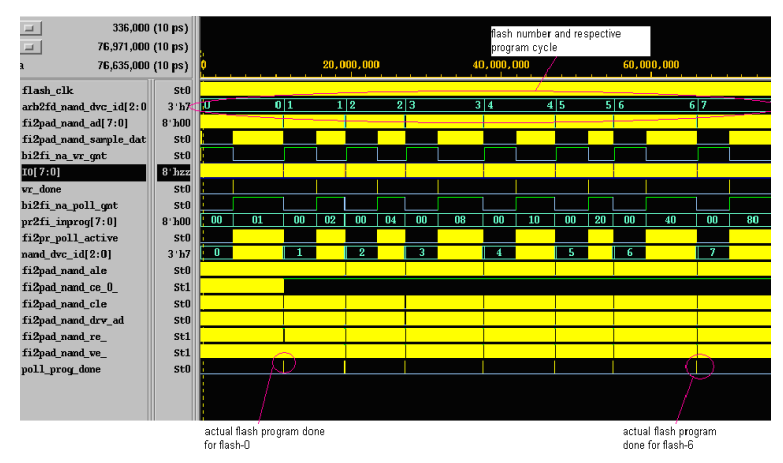

Fig. 8: 8- Flash Programmed

8 NAND flash are programmed serially, one bye one as shown above. Consider typical actual flash program time of 300 usec. 
Time required to program one flash $=300+50=350$ use. Total time required to program 8 NAND Flash $=350 * 8=2800$ usec (approx). Conclusion- More time for actual data write into flash. We can utilize this time to program another flash.

\section{CONCLUSION}

The NAND Flash Controller has only one I/O port and command/address/data multiplexed I/O port in addition to the simple command sets which are provided for the different operations. System uses huge amount of program time for another NAND flash program, so the total program time required for all eight NAND flash is very less as compared to eight individual NAND flash programming.

\section{REFERENCES}

[1] Yet Another Flash File System (YAFFS) http://www.aleph1.co.uk/yaffs/

[2] Journaling Flash File System 2 (JFFS2) http://sourceware.org/jffs $2 /$ http://www.linux-mtd.infradead.org/

[3] Samsung K9K1208U0C 64Mx8/32Mx16 NAND Flash Memory 528 Byte/Page Data Sheet. http://www.samsung.com/Products/Semiconductor/Flash/NAND/51 2Mbit/K9K1208U0C/ds_k9k12xxx0c_rev31.pdf
[4] Samsung K9K2G08U0A 256Mx8/128Mx16/512Mx8 NAND Flash Memory 2112 Byte/Page Data Sheet. http://www.samsung.com/Products/Semiconductor/Flash/NAND/2G bit/K9K2G08U0A/ds_k9k2g08ua_rev02.pdf

[5] Spansion S29GLxxxM MirrorBit Flash Family Data Sheet http://www.spansion.com/datasheets/s29glxxxm_00_b4_e.pdf

[6] Toshiba TC58FVM7T2A 16Mx8 NOR Flash Memory Data Sheet. http://www.toshiba.com/taec/components/Datasheet/58fvm7x2a.pdf

[7] Intel StrataFlash ${ }^{\circledR}$ Embedded Memory (P30) $64 \mathrm{Mb}-1 \mathrm{~Gb}$ Datasheet http://download.intel.com/design/flcomp/datashts/30666601.pdf

[8] AN1823 APPLICATION NOTE Error Correction Code in Single Level Cell NAND Flash Memories http://www.st.com/stonline/books/pdf/docs/10123.pdf

[9] $\mathrm{I}^{2} \mathrm{C}$ Bus Specification Version 2.1 http://www.semiconductors.philips.com/acrobat_download/literature 19398/39340011.pdf

[10] Micron technical note 2906: ECC Module for Xilinx Spartan-3 http://download.micron.com/pdf/technotes/nand/tn2905.pdf

[11] Micron technical note 2908: Hamming Codes for NAND Flash Memories http://download.micron.com/pdf/technotes/nand/tn2908.pdf

[12] NAND Flash Controller IP Core Data Sheet http://www.arasan.com/datasheets/login.php 